\title{
Determining Software Time-to-Market and Testing Stop Time when Release Time is a Change-Point
}

\author{
Ompal Singh \\ Department of Operational Research, \\ University of Delhi, Delhi, India. \\ E-mail: drompalsingh1@gmail.com \\ Saurabh Panwar \\ Department of Operational Research, \\ University of Delhi, Delhi, India. \\ Corresponding author: saurabhpanwar89@yahoo.com \\ P. K. Kapur \\ Amity Center for Interdisciplinary Research, \\ Amity University, Noida, Uttar Pradesh, India. \\ E-mail: pkkapur1@gmail.com
}

(Received April 4, 2019; Accepted September 11, 2019)

\begin{abstract}
In software engineering literature, numerous software reliability growth models have been designed to evaluate and predict the reliability of the software products and to measure the optimal time-to-market of the software systems. Most existing studies on software release time assessment assumes that when software is released, its testing process is terminated. In practice, however, the testing team releases the software product first and continues the testing process for an added period in the operational phase. Therefore, in this study, a coherent reliability growth model is developed to predict the expected reliability of the software product. The debugging process is considered imperfect as new faults can be introduced into the software during each fault removal. The proposed model assumes that the fault observation rate of the testing team modifies after the software release. The release time of the software is therefore regarded as the changepoint. It has been established that the veracity of the performance of the growth models escalates by incorporating the change-point theory. A unified approach is utilized to model the debugging process wherein both testers and users simultaneously identify the faults in the post-release testing phase. A joint optimization problem is formulated based on the two decision criteria: cost and reliability. In order to assimilate the manager's preferences over these two criteria, a multi-criteria decision-making technique known as multi-attribute utility theory is employed. A numerical illustration is further presented by using actual data sets from the software project to determine the optimal software time-to-market and testing termination time.
\end{abstract}

Keywords- Field environment, Imperfect debugging, Multi-attribute utility theory (MAUT), Testing termination time, Software reliability.

\section{Introduction}

System and programming software are pivotal components of computer systems that provide an effective platform to regulate activities and functions among various programs. The successful operation of any application in the computer system chiefly depends on the software systems. Breakdown of these systems may lead to disruption of the necessary information and ultimately project failures. Therefore, it is imperative to control the quality and reliability of the software product during the software development process. Thus, software reliability is one of the prominent aspects of the software development life cycle. Besides, users of the system often expect newly launched software to work successfully without any breakdown. Any instance of failure in the 
International Journal of Mathematical, Engineering and Management Sciences

Vol. 5, No. 2, 208-224, 2020

https://doi.org/10.33889/IJMEMS.2020.5.2.017

software causing catastrophic results could propel them to discontinue it. Consequently, software firms should release products with high reliability to avoid loss of market share, brand value, and long-term gains (Wang et al., 2015).

Software development comprises a series of activities, which are executed, with a risk of fault introduction in the system. Faults occur in the system owing to mistakes and errors made by the developers in performing the software tasks. Therefore, the company gives a lot of attention to the testing process to avoid the introduction of new faults during software development and to eliminate latent faults before delivering the product to users (Kapur et al., 2005). Aside from reliability, the cost function associated with software testing and the debugging process is a decisive attribute for the company. The quality and productivity of the software system depend directly on the cost function. Improving the quality and reliability of the software incurs high development cost; therefore, developers have to attain a trade-off between these two key attributes.

Software failure occurrence is a requisite area of research because it facilitates accurate prediction of software reliability, fault contents, and mean time between failures. In software engineering literature, researchers have offered a great number of software reliability growth models (SRGMs) to predict the failure occurrence phenomenon quantitatively. SRGMs are a mathematical tool that supports developers in measuring the bug content and reliability of the software products. The SRGMs based on non-homogenous process (NHPP) have practical application in the software industry. In NHPP based SRGMs, the mean value function is utilized to evaluate the expected number of failures observed in the software system in a particular time-interval (Kapur et al., 1999). Goel and Okumoto (1979) proposed the most fundamental software reliability growth model that formed the basis of future research. They described the fault detection behavior using the exponential distribution function. Yamada et al. (1983) developed the mathematical model to explicate the failure occurrence phenomenon using delayed S-shaped curve. Some authors have also suggested the models that are flexible and capable of describing both exponential and S-shaped failure pattern (Ohba, 1984). Later, Kapur and Garg (1992) explored the phenomenon of dependent and independent faults and explained the fault detection occurrence using a logistic distribution function. Several other studies were carried out in the last forty years in software reliability engineering to model the software reliability growth function mathematically. Majority of these studies were based on the supposition that the fault debugging process is perfect, i.e., the all the identified faults are removed perfectly from the system without introducing new bugs (Yamada et al., 1983; Kapur et al., 2006). However, this assumption is purely theoretical and has no practical implication (Kapur et al., 2011). Therefore, some researchers have incorporated the concept of imperfect debugging in the modeling the software reliability function (Pham et al., 1999; Xie and Yang, 2003; Kapur et al., 2010, 2011; Roy et al., 2014; Wang et al., 2015; Zhu and Pham, 2018b).

Besides, measuring the fault levels in the software system, another essential feature of the software product is its release time in the market. As the testing resources are finite for every software development project, it is necessary for software developers to know when to stop the testing process and when to deliver the software to its users. Thus, for software firms, the optimal software time-to-market plays a decisive role in achieving long term earnings. In software engineering literature, academicians have suggested various models and policies for evaluating the optimal release time of the software (Kapur et al., 2011). According to the traditional approach, the testing stop time and software release time should coincide, i.e., company should stop the testing process as soon as it delivers the product to its customers (Okumoto and Goel, 1980; Yamada and Osaki, 1987; Dalal and Mallows, 1988; McDaid and Wilson, 2001; Kapur et al., 2014, 2015). However, 
International Journal of Mathematical, Engineering and Management Sciences

Vol. 5, No. 2, 208-224, 2020

https://doi.org/10.33889/IJMEMS.2020.5.2.017

according to some researchers, the software engineers should adopt the policy of releasing the software early and continue the testing process even after the software release to attain the desired reliability aspiration level (Arora et al., 2006; Jiang et al., 2012; Majumdar et al., 2017; Kapur et al., 2019). The benefit of releasing software early is that the competitors will be unable to exploit the market. Moreover, by continuing the testing process in the field environment, testers will be able to achieve the desired level of software reliability and avoid market share loss due to failures in operational phase.

Considering this strategy, the present study formulates a joint optimization problem to determine the optimal software time-to-market and testing termination time. In this paper, the failure occurrence is modeled by dividing the fault debugging process into three phases. The first phase is known as the pre-release phase or testing period of software system wherein developers methodically detect and remove the latent faults from the system. To depict the practical software industry scenario, the debugging process is assumed imperfect, i.e., while removing the observed fault, new faults may arise in the system. This phenomenon is also called error generation. In addition, the probability of completely removing a detected fault is not always one. The second phase of the developed model is termed as the post-release phase. This phase starts at a release time of the software and ends when the developers terminate the testing process. During this phase, both users and testers observe the failures in the system. Users on encountering a software failure will report it to the testing team who then immediately remove the fault from the system. On correcting the fault, the developers send patches to its users for updating the software system. After a fixed time-interval, testers should stop the testing process as prolong testing will result in high development cost. However, when testers terminate the testing process, the users continue observing the faults and reporting it to the testers. Therefore, in the third phase, post-testing phase users detect the bugs and testing team correct the faults.

Further, to avoid failures at the user's end, the developers modify its fault detection intensity in the post-release phase. Therefore, the release of the software is considered as a change-point. The timepoint at which the fault detection rate alters is termed as a change-point (Kapur et al., 2008). Besides, the new release time policy with field-testing (FT) phase is deduced and compared with the traditional release time policy with no-field testing (NFT) phase. For optimization purpose, two key attributes, namely, reliability indicator and the cost function is considered. To determine a trade-off between these two attributes, multi-attribute utility theory (MAUT), a decision-making approach is employed. A practical implication of the study is provided using a numerical illustration.

\section{Model Development}

In this section, a unified approach is employed to model the software reliability growth process. A generalized SRGM is suggested to quantify the faults debugging phenomenon during the entire product lifecycle. In the proposed framework, it is assumed that the software developers continue the testing process of the software for a specific period even after its market-entry. Therefore, the fault debugging process is summarized into three phases, namely, pre-release testing phase, postrelease testing phase and post-testing phase. These phases are graphically depicted in Figure 1. Moreover, in factual testing scenario, the debugging process can never be perfect, i.e. not all detected faults will be completely removed from the system. This can be due to tester's proficiency level and faults characteristics (Zhu and Pham 2018a). In addition, some faults that were not present in the system earlier may become apparent after the debugging process. Therefore, in the present study, the debugging process is considered imperfect as some new bugs may be induced in the 
International Journal of Mathematical, Engineering and Management Sciences

Vol. 5, No. 2, 208-224, 2020

https://doi.org/10.33889/IJMEMS.2020.5.2.017

system during fault removal process.

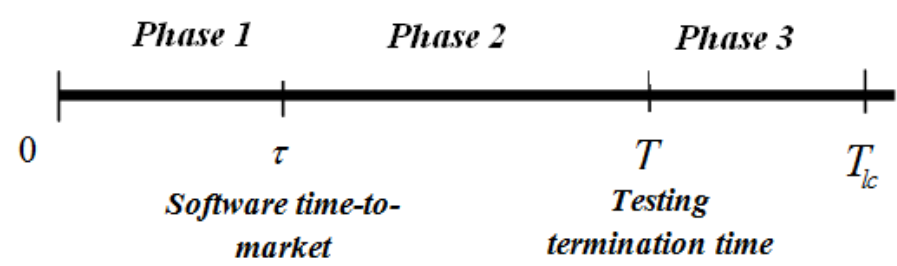

Figure 1. Different phases of failure occurrence phenomenon

\subsection{NHPP based Software Reliability Growth Model}

In the present paper, the Non-homogeneous Poisson Process (NHPP) has been utilized to illustrate the failure phenomenon of the software system. Software Reliability Growth Models (SRGMs) based on NHPP assumes that the software failure occurs randomly, which occurs due to the faults present in the software system. The counting process $\{N(t), t \geq 0\}$ of the NHPP depicts the total of number of failures by the execution time $t$ and it is defined as:

$\operatorname{Pr}[N(t)=k]=\frac{[m(t)]^{k}}{k !} \cdot e^{-m(t)}, k=0,1,2 \ldots$

where $m(t)$ is represents the expected number of faults discovered by time $t$ or the mean value function. It is evaluated as:

$m(t)=\int_{0}^{t} \theta(u) d u$

where, $\theta(u)$ is the fault intensity function.

\subsection{Notations}

This subsection lists all the notations used in the modeling framework.

$\tau \quad$ The release time of the software, which is also a change-point

$m_{t_{1}}(t) \quad$ Mean value function of fault detected in pre-release phase

$m_{t_{2}}(t-\tau)$ Mean value of faults identified by testing team in post-release phase

$m_{u_{1}}(t-\tau) \quad$ Mean value function of faults detected by the users post-release phase

$m_{u_{2}}(t-T)$ Mean value function of faults identified by the users in post-testing phase

$F_{t_{1}}(t) \quad$ Failure observation distribution function of the tester before change-point $\tau$

$F_{t_{2}}(t) \quad$ Failure observation distribution function of the tester after change-point $\tau$

$F_{u}(t-\tau) \quad$ Failure observation distribution function of the users

$a \quad$ Initial fault content level in the software system

$T \quad$ Testing duration of the software product; $T>\tau$ 
International Journal of Mathematical, Engineering and Management Sciences

Vol. 5, No. 2, 208-224, 2020

https://doi.org/10.33889/IJMEMS.2020.5.2.017
$T_{l c}$
Fixed duration of software lifecycle
$C_{1}$
Cost of testing the software per unit time in the testing period
$C_{2}$
Market opportunity cost, which is a function of software release time
$\mathrm{C}_{3}$
Cost of removing the observed bugs in pre-release phase
$C_{4}$
Cost of removing the bugs observed by the testers in post-release phase
$C_{5}$
Cost of removing the bugs observed by the users in post-release phase.
$C_{6}$
Cost of removing the bugs identified by the users in the post-testing phase

\subsection{Assumptions}

The growth models for fault detection phenomenon are based upon the following assumptions:

(i) The software fault removal phenomenon is based on the Non-homogenous Poison Process (NHPP).

(ii) Every failure in the software is independently and identically distributed over the lifecycle of the system.

(iii) The time between fault detection and removal is negligible.

(iv) The bugs are not debugged perfectly upon identification.

(v) The fault removal process may induce new bugs in the software system with rate $\alpha$.

(vi) When a software failure occurs, an instantaneous repair effort starts and the following may occur:

- Fault content is reduced by one with probability $p$.

- Fault content remains unchanged with probability $(1-p)$.

(vii) After the release of the software, the users also contribute in fault detection process.

(viii) As soon as users identify the faults, they will report it to developers for removing it. After detecting the faults, customers immediately report it to the testing team. Testers then send a patch to users once they rectify the problem.

(ix) After successful fault debugging process during the post-release testing period, the developers will provide a patch to their users for updating the system. The cost of security patch is assumed negligible.

\subsection{Generalized Imperfect Debugging based SRGM}

\subsubsection{Phase 1: Pre-Release Testing Phase $[0, \tau)$}

Before releasing software in the market, testing team rigorously identifies and removes the bugs lying dormant in the system. It is considered that the expected number of faults removed at any time is directly proportional to the bugs present in the system. An NHPP based software reliability model under imperfect debugging is formulated as:

$\frac{d m_{t_{1}}(t)}{d t}=p \frac{f_{t_{1}}(t)}{1-F_{t_{1}}(t)}\left(a+\alpha_{1} m_{t_{1}}(t)-m_{t_{1}}(t)\right)$

where $\frac{f_{t_{1}}(t)}{1-F_{t_{1}}(t)}$ denotes the hazard rate of fault detection in pre-release testing period, $p$ is the probability of perfect debugging and $\alpha_{1}$ is the rate of error generation. 
International Journal of Mathematical, Engineering and Management Sciences

Vol. 5, No. 2, 208-224, 2020

https://doi.org/10.33889/IJMEMS.2020.5.2.017

On substituting the initial condition in equation (3), $t=0, m_{t_{1}}(t)=0$, the expected number of bugs removed by the testers during pre-release testing phase is obtained as:

$m_{t_{1}}(t)=\frac{a}{1-\alpha_{1}}\left[1-\left(1-F_{t_{1}}(t)\right)^{p\left(1-\alpha_{1}\right)}\right]$

Further, if fault distribution function follows an exponential function, then the expected number of bugs removed in pre-release phase is given as:

$m_{t_{1}}(t)=\frac{a}{1-\alpha_{1}}\left(1-e^{-b_{1} p\left(1-\alpha_{1}\right) t}\right)$

where, $b_{1}$ is the rate parameter denoting the fault detection rate. Equation (5) represents the mean value function of faults debugging produces in time interval $[0, \tau)$.

\subsubsection{Phase 2: Post-Release Testing Phase $[\tau, T)$}

After the software is avaliable to the customers, they also assist developers in identifying the faults during this phase. Therefore, during post-release testing phase, both the testing team and users thoroughly identify faults from the software system. After pre-release phase, the faults that are remained undetected in the software are simultaneously identified by both the testers and users. Therefore, it is assumed a fixed fraction $(\lambda)$ of remaining faults will be detected by testers and $(1-\lambda)$ fraction of leftover faults from phase 1 will be detected by the users. In addition, as the software is already in the field environment, therefore, comapany will modify their tetsing strategy to accelerate the debugging process. Correspondingly, testers will alter their fault detection rate. As a result, the release time of the software $(\tau)$ will also act as a change-point for the developer's fault removal rate.

\section{Case 1: Fault Detection by Testing Team}

The mean value function of faults discovered by the testing team during post-testing phase becomes:

$\frac{d m_{t_{2}}(t-\tau)}{d t}=p \frac{f_{t_{2}}(t)}{1-F_{t_{2}}(t)}\left(\begin{array}{l}\lambda a *\left[1-F_{t_{1}}(\tau) *\right]+\alpha_{2} m_{t_{2}}(t-\tau) \\ -m_{t_{2}}(t-\tau)\end{array}\right)$

where, $a^{*}=\frac{a}{1-\alpha_{1}} ; \quad F_{t_{1}}(\tau)^{*}=\left[1-\left(1-F_{t_{1}}(t)\right)^{p\left(1-\alpha_{1}\right)}\right]$.

In equation (6), $\frac{f_{t_{2}}(t)}{1-F_{t_{2}}(t)}$ denotes the fault detection rate of the testers after change-point $\tau$ and $\alpha_{2}$ denotes the bug introduction rate during this phase. Using the initial condition, $t=\tau, m_{t_{2}}(t)=0$, the above differential equation can be further solved to obtain the following closed-form solution:

$m_{t_{2}}(t-\tau)=\lambda a^{* *}\left(1-F_{t_{1}}(\tau)\right)^{p\left(1-\alpha_{1}\right)}\left[1-\left(\frac{1-F_{t_{2}}(t)}{1-F_{t_{2}}(\tau)}\right)^{p\left(1-\alpha_{2}\right)}\right]$ 
International Journal of Mathematical, Engineering and Management Sciences

Vol. 5, No. 2, 208-224, 2020

https://doi.org/10.33889/IJMEMS.2020.5.2.017

where, $a^{* *}=\frac{a}{\left(1-\alpha_{1}\right)\left(1-\alpha_{2}\right)}$.

Further, it is assumed that the fault discovery process follows an exponential distribution function, therefore, the expected bugs detected by the testing team in interval $[\tau, T)$ is given as:

$m_{t_{2}}(t-\tau)=\lambda \frac{a e^{-b_{1} p\left(1-\alpha_{1}\right) \tau}}{\left(1-\alpha_{1}\right)\left(1-\alpha_{2}\right)}\left(1-e^{-b_{2} p\left(1-\alpha_{2}\right)(t-\tau)}\right)$

where, $b_{2}$ is the fault detection rate parameter after change-point $\tau$.

\section{Case 2: Fault Detection by Users}

When users execute instructions on the software system, they may experience failure due to the faults present in the device. They report it the testing team who instantly start the debugging process. Thus, the differential equation representing the users bug detection rate is given as:

$\frac{d m_{u_{1}}(t-\tau)}{d(t-\tau)}=p \frac{f_{u}(t-\tau)}{1-F_{u}(t-\tau)}\left(\begin{array}{l}(1-\lambda) a *\left[1-F_{t_{1}}(\tau)^{*}\right]+\alpha_{2} m_{u_{1}}(t-\tau) \\ -m_{u_{1}}(t-\tau)\end{array}\right)$

where, $a^{*}=\frac{a}{1-\alpha_{1}} ; F_{t_{1}}(\tau)^{*}=\left[1-\left(1-F_{t_{1}}(t)\right)^{p\left(1-\alpha_{1}\right)}\right]$.

In equation (9), signifies the hazard rate of fault detection rate of the users. Further, using the initial condition, that at $t=\tau, m_{u_{1}}(t-\tau)=0$, the expected number of faults discovered by the users is obtained:

$m_{u_{1}}(t-\tau)=(1-\lambda) a^{* *}\left(1-F_{t_{1}}(\tau)^{p\left(1-\alpha_{1}\right)}\left[1-\left(1-F_{u}(t-\tau)\right)^{p\left(1-\alpha_{2}\right)}\right]\right.$

where, $a^{* *}=\frac{a}{\left(1-\alpha_{1}\right)\left(1-\alpha_{2}\right)}$.

As fault discovery process is assumed to depict exponential distribution function, therefore, the mean value function of faults detected by users becomes:

$m_{u_{1}}(t-\tau)=(1-\lambda) \frac{a e^{-b_{1} p\left(1-\alpha_{1}\right) \tau}}{\left(1-\alpha_{1}\right)\left(1-\alpha_{2}\right)}\left(1-e^{-b_{3} p\left(1-\alpha_{2}\right)(t-\tau)}\right)$

where, $b_{3}$ is the fault detection rate parameter of customers in post-release testing phase.

\subsubsection{Phase 3: Post-Testing Phase $\left[T, T_{l c}\right]$}

In this study, it is assumed that testers terminate their testing process after a fixed time interval. Let $T$ be the time-point at which developers stop the testing process. Beyond time-point $T$, only users will identify bugs that are remaining in the system. During this phase, users will notify the testers about the faults discovered by them, who then make an effort to remove it immediately. Thus, the 
International Journal of Mathematical, Engineering and Management Sciences

Vol. 5, No. 2, 208-224, 2020

https://doi.org/10.33889/IJMEMS.2020.5.2.017

expected number of faults detected at any instant of time in interval $\left[T, T_{l c}\right]$ is given as:

$\frac{d m_{u_{2}}(t-\tau)}{d(t-\tau)}=p \frac{f_{u}(t-\tau)}{1-F_{u}(t-\tau)}\left(Z_{a}+\alpha_{2} m_{u_{2}}(t-\tau)-m_{u_{2}}(t-\tau)\right)$

where, $Z_{a}=a(T)-m_{t_{1}}(\tau)-m_{t_{2}}(T-\tau)-m_{u_{1}}(T-\tau)$ is the remaining faults in the system after timepoint $T$, i.e.

$Z_{a}=\frac{a}{\left(1-\alpha_{1}\right)}\left(1-F_{t_{1}}(\tau)\right)^{p\left(1-\alpha_{1}\right)}\left(\begin{array}{c}\left(1-\alpha_{1}\right)-\lambda\left(1-\left(\frac{1-F_{t_{2}}(T)}{1-F_{t_{2}}(\tau)}\right)\right)^{p\left(1-\alpha_{2}\right)} \\ -(1-\lambda)\left[1-\left(1-F_{u}(T-\tau)\right)^{p\left(1-\alpha_{2}\right)}\right]\end{array}\right)$

and $a(T)=a+\alpha_{1} m_{t_{1}}(\tau)+\alpha_{2} m_{t_{2}}(T-\tau)+\alpha_{2} m_{u_{1}}(T-\tau)$.

Using the boundary condition that at $t=T, m_{u_{2}}(t-\tau)=0$, the analytical solution of equation (12) can be given as:

$m_{u_{2}}(t-\tau)=Z_{a}\left[1-\left(\frac{1-F_{u}(t-\tau)}{1-F_{u}(T-\tau)}\right)^{p_{1}\left(1-\alpha_{2}\right)}\right]$

When fault observation phenomenon occurs with exponentially decreasing rate, then the mean value function of fault identification for the users in phase 3 takes the following form:

$m_{u_{2}}(t-\tau)=\frac{a}{\left(1-\alpha_{1}\right)} e^{-b_{1} p_{1}\left(1-\alpha_{1}\right) \tau}\left(\begin{array}{c}\left(1-\alpha_{1}\right)-\lambda\left(1-e^{-b_{2} p_{1}\left(1-\alpha_{2}\right)(T-\tau)}\right) \\ -(1-\lambda)\left(1-e^{-b_{3} p_{1}\left(1-\alpha_{2}\right)(T-\tau)}\right)\end{array}\right)\left(1-e^{-b_{3} p_{1}\left(1-\alpha_{2}\right)(t-T)}\right)$

Equation (15) provides the expected number of bugs discovered by the users in time interval $\left[T, T_{l c}\right]$.

\subsubsection{Proposed Cost Model}

In the present research, cost model comprises of following cost functions:

(i) Testing Cost: This cost refers to per unit testing cost and is associated with the tester's activities such as such as test planning, test case generation, test execution, and analysis of testing results. In software engineering literature, testing cost has been established as a linear function of testing time (Kapur et al., 2019). Therefore, its functional form is given as:

$C_{\text {Testing }}(t)=C_{1} T$

(ii) Market Opportunity Cost: The market opportunity is the loss incurred by the company when the release of the software is delayed and correspondingly market is captured by the competitive 
International Journal of Mathematical, Engineering and Management Sciences

Vol. 5, No. 2, 208-224, 2020

https://doi.org/10.33889/IJMEMS.2020.5.2.017

firms. Market opportunity cost is the increasing function of the release time of the software. It is a crucial parameter to take into consideration for decision makers as it assists in examining the market manipulation by the competitors. In the past literature, the market opportunity cost is considered as a quadratic function of the software time-to-market (Jiang et al., 2012). Therefore, following functional form is used in the present study:

$$
C_{\text {Market_opp }}(t)=C_{2} \tau^{2}
$$

(iii) Cost of Faults Removal in Pre-Release Testing Phase: This attribute comprises direct cost associated with detecting and debugging the faults during the time interval $[0, \tau)$. It is assumed a linear function of the expected number of faults discovered during this period. Its functional form is given as:

$$
C_{\text {pre-release }}(t)=C_{3} m_{t_{1}}(\tau)
$$

(iv) Cost of Faults Removal in Post-Release Testing Phase: After the release of the software at $\tau$, both testers and customers identify the faults present in the system. Therefore, the cost function during this phase is divided into two components. First component describes the cost of debugging faults that were detected by the testers. The second component represents the cost of faults debugging, which were identified by the users and then removed by the testing team. Therefore, the functional form of the debugging cost during post-release testing phase becomes:

$$
C_{\text {post-release }}(t)=C_{4} m_{t_{2}}(T-\tau)+C_{5} m_{u_{1}}(T-\tau)
$$

(v) Cost of Faults Removal in Post-Testing Phase: After the testers stops the testing process at time $T$, users may still encounter failure of the software system due to the bugs that were remained undetected in the previous phases. Thus, users identify these bugs and immediately report it to the developers who then quickly remove them. Therefore, the cost of debugging the faults detected during period $\left[T, T_{l c}\right]$ is given as:

$$
C_{\text {post-testing }}(t)=C_{6} m_{u_{2}}\left(T_{l c}-T\right)
$$

Therefore, the total cost structure when field-testing is considered is given as:

$$
C(\tau, T)=C_{1} T+C_{2} \tau^{2}+C_{3} m_{t_{1}}(\tau)+C_{4} m_{t_{2}}(T-\tau)+C_{5} m_{u_{1}}(T-\tau)+C_{6} m_{u_{2}}\left(T_{l c}-T\right)
$$

Now, in the conventional approach, $\tau^{*}=T^{*}$, i.e. testing stop time and software release time coincides. Then, the cost structure in no-field testing scenario will be:

$C(\tau)=C_{1} \tau+C_{2} \tau^{2}+C_{3} m_{t_{1}}(\tau)+C_{6} m_{u_{2}}\left(T_{l c}-\tau\right)$

\section{Optimization Model using Multi-Attribute Utility Theory}

The objective of the present study is to optimize software time-to-market and optimal testing duration based on the cost-efficiency and reliability measures by considering the software release time as change point for tester's fault detection rate. Further, the proposed release time policy is 
International Journal of Mathematical, Engineering and Management Sciences

Vol. 5, No. 2, 208-224, 2020

https://doi.org/10.33889/IJMEMS.2020.5.2.017

compared with the traditional release time policy where no-field testing is involved. Therefore, two optimization problems are formulated using the Multi-attribute utility theory (MAUT). MAUT is a multi-criteria decision-making (MCDM) approach used for solving the optimization problem considering multiple attributes with conflicting objectives (Keeney, 1971). Li et al. (2011) was the first to incorporate this MCDM technique in the research field of software reliability. MAUT comprises of following steps:

Step 1: Identify the appropriate factors for the study under consideration.

Step 2: Formulate the single attribute utility function (SAUF) for each factor.

Step 3: Estimate the weight parameters for each factor involved in the study.

Step 4: Develop the multi-attribute utility function (MAUF).

For the present study, the optimization problem can be explained using these four steps.

\subsection{Selection of Attributes}

The critical factors that have the most relevance to the study should be considered in the optimization model. These attributes should be quantifiable in a meaningful and practical way. The principal concern of the software firms is to provide software to its users that are both reliable and secure. Therefore, maximizing the reliability of the software product is the prime objective of the decision makers when assessing the release time and testing duration of the system. A most common indicator of reliability of a software system is the ratio of the expected number of bugs removed by time $t$ to the total fault content present in the software (Li et al., 2011). Thus, the first factor included in the present study is:

For field-testing (FT) policy:

Maximize $R=\frac{m(\tau, T)}{a(T)}$, i.e.

Maximize $R=\frac{m_{t_{1}}(\tau)+m_{t_{2}}(T-\tau)+m_{u_{1}}(T-\tau)}{a+\alpha_{1} m_{t_{1}}(\tau)+\alpha_{2}\left(m_{t_{2}}(T-\tau)+m_{u_{1}}(T-\tau)\right)}$

For no-field testing (NFT) policy

Maximize $\mathrm{R}=\frac{m(\tau)}{a(\tau)}=\frac{m_{t_{1}}(\tau)}{a+\alpha_{1} m_{t_{1}}(\tau)}$

where, $R$ is a reliability indicator used as a key factor in MAUT. It value ranges from $0 \leq R \leq 1$. As the reliability is an increasing function of time, it reaches the maximum value when time goes to infinity.

The second critical factor is the cost function associated with testing and debugging process. The software firm wants to improve the quality of its product but at the minimal cost. Therefore, for decision makers, the analysis of cost budget consumption plays an important role. Thus, the second attribute cost function is given as: 
International Journal of Mathematical, Engineering and Management Sciences

Vol. 5, No. 2, 208-224, 2020

https://doi.org/10.33889/IJMEMS.2020.5.2.017

Under field-testing (FT) policy:

minimize $C=\frac{C(\tau, T)}{C_{b}}$

where, $C(\tau, T)=C_{1} T+C_{2} \tau^{2}+C_{3} m_{t_{1}}(\tau)+C_{4} m_{t_{2}}(T-\tau)+C_{5} m_{u_{1}}(T-\tau)+C_{6} m_{u_{2}}\left(T_{l c}-T\right)$ and $C_{b}$ is the companies budget allocated for testing and debugging process.

Under no field-testing (NFT) policy:

minimize $C=\frac{C(\tau)}{C_{b}}$

where, $C(\tau)=C_{1} \tau+C_{2} \tau^{2}+C_{3} m_{t_{1}}(\tau)+C_{6} m_{u_{2}}\left(T_{l c}-\tau\right)$

\subsection{Evaluate SAUF}

In MAUT, utility functions are used to depict goal of each factor. In literature, two functional forms of utility function are commonly used: linear and exponential type (Kapur et al., 2019). The general form of linear function is $u(x)=l+m x$ and of exponential is $u(x)=l+m e^{p x}$. For a particular study, the functional form is determined using the interviews with the experts, survey or through lottery. For the current research, linear form has been utilized based on the opinion of software experts and analysts. Thus, the utility function of two factors, namely, reliability indicator and cost function is:

$u(C)=l_{c}+u_{c} C$ and $u(R)=l_{r}+m_{r} R$

In addition, the utility function is bounded with the best, $u\left(x^{\text {best }}\right)=1$ and the worst, $u\left(x^{\text {worst }}\right)=0$ case scenario for each factor under consideration. Further, the bounds are measured based on the managers and decision maker's aspirations:

a) For the reliability indictor factor, minimum $60 \%$ of the faults must be detected and maximum aspiration level is $100 \%$.

b) For cost factor, the minimum budget requirement is $60 \%$ and the maximum obligation is $100 \%$.

Therefore, the bounds for each factor is: $C^{\text {worst }}=0.6, C^{\text {best }}=1, R^{\text {worst }}=0.6$ and $R^{\text {best }}=1$. Using these boundary conditions, the SAUF for the two attributes will the following functional form:

$U(C)=2.5 C-1.5$ and $U(R)=2.5 R-1.5$

\subsection{Assign Weight Parameters to Each Attribute}

The weight parameter signifies the relative importance of an attribute over other attributes in the optimization problem. When number of factors under consideration is small, lottery method or management's discretion is suggested. As in the present paper, only two factors have been utilized, the priority to the factor is assigned on the management's judgment. According to software development managers, more importance should be given to the reliability indicator. Consequently, weight given to reliability indicator is $w_{R}=0.6$. Additionally, the sum of the weight parameters 
International Journal of Mathematical, Engineering and Management Sciences

Vol. 5, No. 2, 208-224, 2020

https://doi.org/10.33889/IJMEMS.2020.5.2.017

should be 1 . Thus, the weight assigned to cost factor is $w_{c}=0.4$.

\subsection{Formulation of MAUF}

Multi-attribute utility function is the arithmetic sum of all the single utility functions (SAUF). Thus, the additive form of MAUF for the present study is given as:

$\operatorname{Max} U(R, C)=w_{R} U(R)-w_{C} U(C)$

where $w_{R}+w_{c}=1$ and $U(R)$ and $U(C)$ describes the single utility functions for factor reliability indicator and cost respectively.

The objective of the software developers is to maximize the reliability indicator and minimize the cost function. Therefore, to reflect the aim of minimizing the cost attribute, negative assign is multiplied with cost component. On solving the above maximization problem, the optimal values of decision variables $\tau^{*}$ and $T *$ will be obtained. From substituting the values from previous steps, following functional form is attained:

$\operatorname{Max} U(R, C)=0.6 \times(2.5 R-1.5)-0.4 \times(2.5 C-1.5)$

where

$w_{R}+w_{c}=1$ and $\frac{C(\tau, T)}{C_{b}} \leq 1$

\section{Numerical Illustration}

The estimation efficiency and prediction accuracy of the developed SRGM is asserted using the actual fault count data of Tandem computers, which has been collected for the pre-release testing period. Tandem computers data consists of four successive release failure data (Wood, 1996). As the first release data follows an exponential growth pattern, therefore, for the current problem, the first release data has been utilized. In the first release failure data, 100 faults were debugged in 20 weeks of testing period. The parameters estimation is carried-out using the nonlinear least square (NLLS) regression technique (Marquardt, 1963). The complete empirical analysis is executed using the software package known as SAS (SAS/ETS User's Guide, 2004). The estimated values of the model parameters are: $a=107.65, b_{1}=0.55205, p=0.185, \alpha_{1}=0.15905$. To examine the prediction capability of the model, two statistical measures, namely root mean square error (RMSE) and R-square are determined. The values of goodness-of-fit measures are RMSE $=3.607$ and $R^{2}=$ 0.986. The graphical depiction of actual versus predicted fault count is shown in Figure 2. 
International Journal of Mathematical, Engineering and Management Sciences

Vol. 5, No. 2, 208-224, 2020

https://doi.org/10.33889/IJMEMS.2020.5.2.017

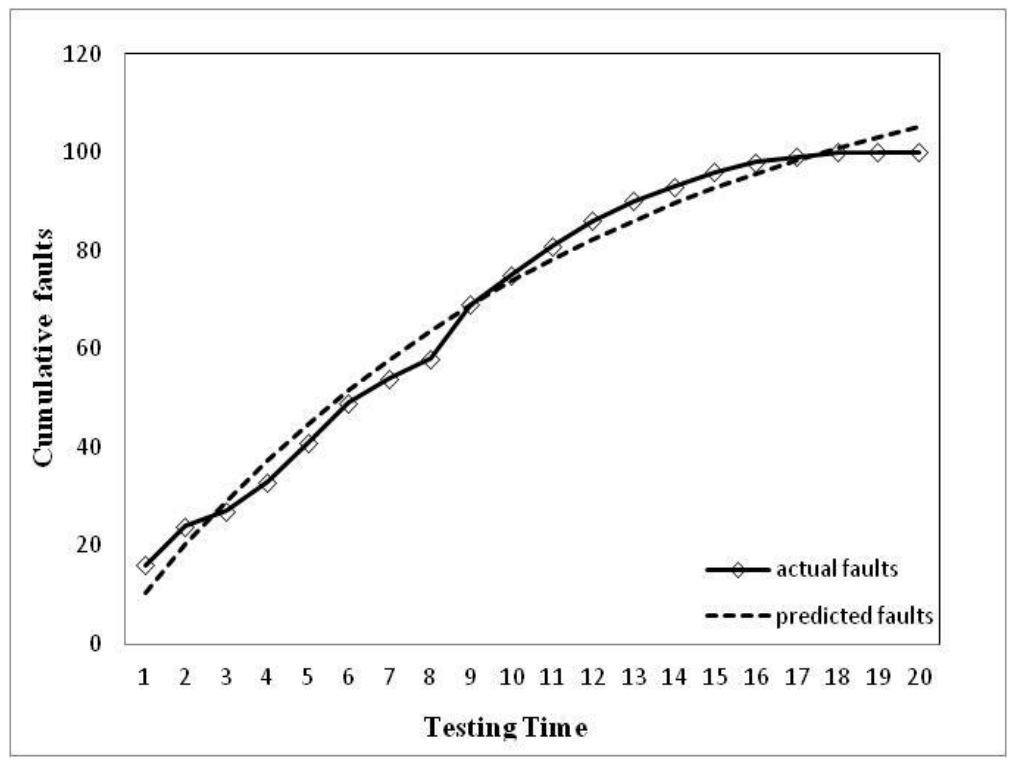

Figure 2. Actual versus predicted fault count of Tandem computers first release

The parameters of post-release and post-testing periods are set based on the software managers judgement. The management team considered that the testers have improved the detection rate of the faults by $50 \%$ after change-point $\tau$. In addition, the users bug detection effectiveness is only $60 \%$ of that of the developers. Besides, testers are more efficient and proffessional than useres therefore, it is assumed that $\lambda=0.6$ i.e. $60 \%$ of the reaming faults will be detected by the testting team and rest will identify by th users. Moreover, the cost parameters are assumed based on the prior literature. The parameters values used in the optimization study are summarized in Table 1.

Further, the formulated MAUF is evaluated using the above parameters values. For solving the optimization model, software tool known as MAPLE is employed. The goal of the proposed optimization problem is to determine the optimal software time-to-market and testing duration that will maximize the utility function. The optimal results for both the release policy are listed in Table 2 . The concavity of the developed optimization problem is established using the pictorial representation of the utility function under field-testing and no field-testing release time policy as shown in Figure 3 and 4 respectively.

Table 1. Parameter values

\begin{tabular}{|c|c|c|c|}
\hline Parameters & Values & Parameters & Values \\
\hline$b_{2}$ & 0.88328 & $C_{5}$ & $\$ 160$ \\
\hline$b_{3}$ & 0.22082 & $C_{6}$ & $\$ 180$ \\
\hline$C_{1}$ & $\$ 100$ & $C_{\mathrm{b}}$ & 100 weeks \\
\hline$C_{2}$ & $\$ 10$ & $T_{l c}$ & 0.6 \\
\hline$C_{3}$ & $\$ 40$ & $\lambda$ & 0.15905 \\
\hline$C_{4}$ & $\$ 70$ & $\alpha_{2}$ & \\
\hline
\end{tabular}


International Journal of Mathematical, Engineering and Management Sciences

Vol. 5, No. 2, 208-224, 2020

https://doi.org/10.33889/IJMEMS.2020.5.2.017

Table 2. Optimal results

\begin{tabular}{|l|c|c|c|}
\hline Release Policies & $\boldsymbol{U}\left(\tau^{*}, \boldsymbol{T}^{*}\right)$ & $\tau^{*}$ (in weeks) & $\boldsymbol{T} *($ in weeks) \\
\hline Field-testing (FT) policy & 0.662 & 10.269 & 38.668 \\
\hline No field-testing (NFT) policy & 0.463 & 24.123 & - \\
\hline
\end{tabular}

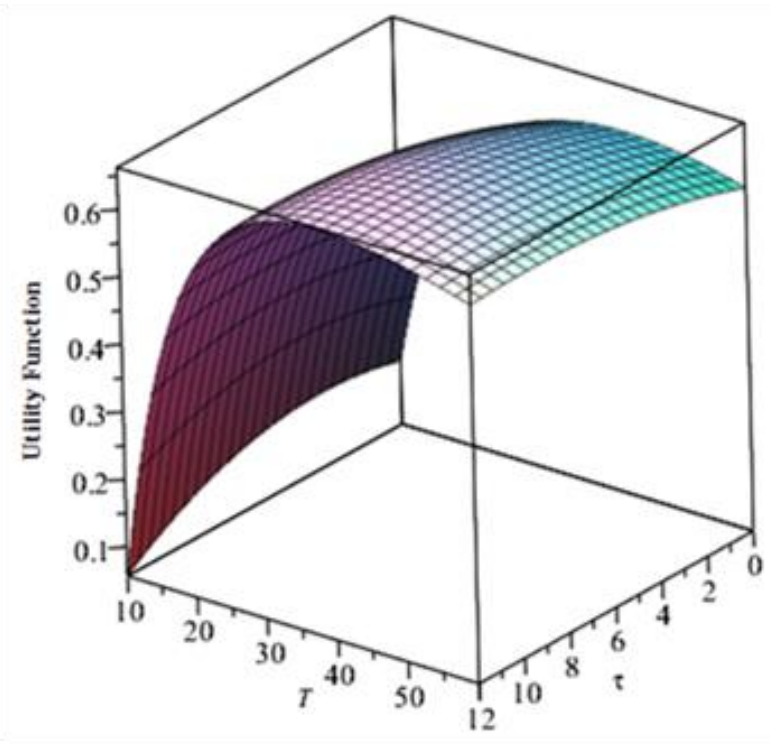

Figure 3. Concavity plot under field-testing (FT) release time policy

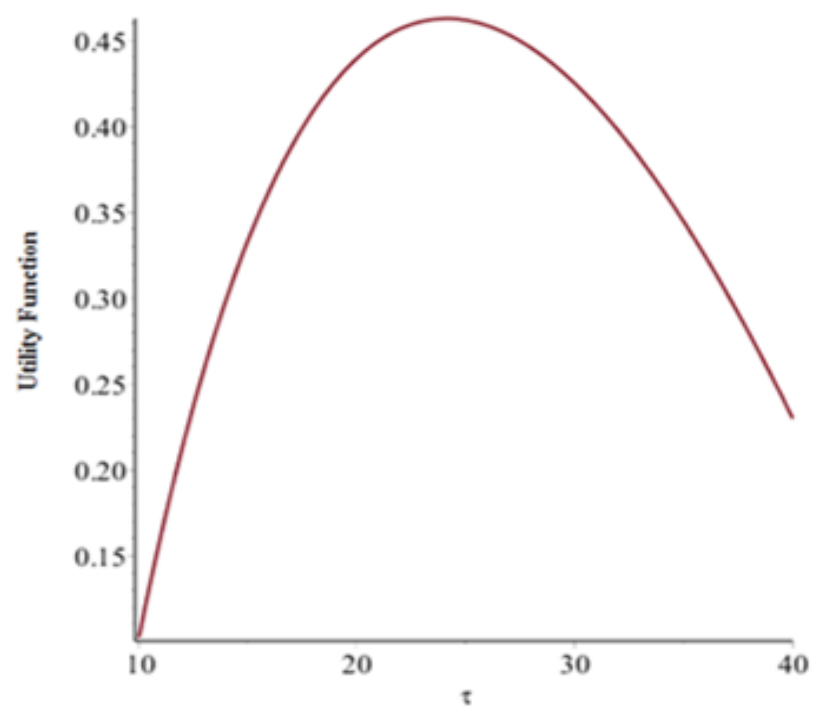

Figure 4. Concavity plot under no field-testing (NFT) release time policy 
International Journal of Mathematical, Engineering and Management Sciences

Vol. 5, No. 2, 208-224, 2020

https://doi.org/10.33889/IJMEMS.2020.5.2.017

When company adopts the strategy to continue the testing process for an added period in the field environment, more utility for reliability and cost measure is attained. Under field-testing policy, $66.2 \%$ utility is obtained when software firm releases the software at $\tau^{*}=10.012$ weeks after the initialization of the testing process. Moreover, company should continue the testing process for next 28.399 weeks to improve the reliability of the system. Therefore, developers should terminate the testing process at $T^{*}=38.668$ weeks. By doing so, the decision makers will be able to achieve the aspiration level of the reliability and cost attributes. However, if the company follows the traditional policy of terminating the testing process at the release time of the software, then only $46.3 \%$ utility can be achieved with $\tau^{*}=24.123$ weeks.

\section{Conclusion}

This paper explores the release time problem for software products. The optimization problem developed in the present study considers release time and testing stop time as two different decision variables. A failure occurrence behavior is modeled in three phases: pre-release or system testing phase, post-release or field-testing phase, and post-testing phase. During the pre-release testing phase, testers thoroughly identify and debug faults to maintain the reliability of the system before releasing the software in the market. It is considered that the change-point in the fault detection rate of testers occurs at the release time of the software. This is because, after the release of the software, testers want to decrease the failure in the operational phase and therefore, accelerates its debugging process in the field-testing period. During this phase, users also contribute to the fault observation process. However, the combined rate of user's fault detection is less as compared to the professional testing team. As the testing team has limited resources, therefore, they terminate the testing process after a certain time when the desired level of reliability is attained after the release of the software. Consequently, in the third phase, the post-testing phase only users observe the faults and report the error to the testing team who then immediately make efforts to remove them. Thus, the fault detection process continues until the software lifecycle.

In the present paper, the fault detection process is modeled using the unified approach and exponential distribution function is further applied to describe the fault observation phenomenon in all three phases. Additionally, a joint optimization problem is developed to determine the optimal time-to-market and testing stop time. To obtain the optimal results, two essential software development criteria, specifically, reliability indicator and the cost function is taken into consideration. Further, to achieve a trade-off between these two conflicting criteria, a decisionmaking technique called multi-attribute utility theory (MAUT) is applied. The actual fault content data of Tandem computers are utilized to estimate the model parameters and solve the optimization problem. A numerical example is also presented to analyze the practical relevance of the proposed release time policy. The optimal results of the developed release time policy are further compared with the conventional release time policy wherein release time and testing stop time is characteristically considered as one decision variable. Findings of the present research provide vital managerial insights for the software managers and demonstrate to release the software early in order to capture the maximum market share and continue to the testing process for an additional period in the operational phase to avoid failures at the user end. In addition, the proposed study can be further extended by incorporating the effect of testing efforts on the faults detection process. Moreover, in future, the reliability attribute can be evaluated using the software reliability function, which describes the conditional probability that software will perform without failure in a given time interval. 
International Journal of Mathematical, Engineering and Management Sciences

Vol. 5, No. 2, 208-224, 2020

https://doi.org/10.33889/IJMEMS.2020.5.2.017

\section{Conflict of Interest}

The authors declare that there is no conflict of interest for this publication.

\section{Acknowledgment}

The research work presented in this paper is supported by grants to the first author from DST via DST PURSE phase II, India.

\section{References}

Arora, A., Caulkins, J.P., \& Telang, R. (2006). Research note-sell first, fix later: impact of patching on software quality. Management Science, 52(3), 465-471.

Dalal, S.R., \& Mallows, C.L. (1988). When should one stop testing software? Journal of the American Statistical Association, 83(403), 872-879.

Goel, A.L., \& Okumoto, K. (1979). Time-dependent error-detection rate model for software reliability and other performance measures. IEEE Transactions on Reliability, 28(3), 206-211.

Jiang, Z., Sarkar, S., \& Jacob, V.S. (2012). Postrelease testing and software release policy for enterpriselevel systems. Information Systems Research, 23(3-part-1), 635-657.

Kapur, P.K., \& Garg, R.B. (1992). A software reliability growth model for an error-removal phenomenon. Software Engineering Journal, 7(4), 291-294.

Kapur, P.K., Gupta, A., \& Singh, O. (2005). On discrete software reliability growth model \& categorization of faults. Opsearch, 42(4), 340-354.

Kapur, P.K., Gupta, A., Yadavalli, V.S.S., \& Claasen, S.J. (2006). Flexible software reliability growth models. South African Journal of Industrial Engineering, 17(2), 109-125.

Kapur, P.K., Khatri, S.K., Tickoo, A., \& Shatnawi, O. (2014). Release time determination depending on number of test runs using multi attribute utility theory. International Journal of System Assurance Engineering and Management, 5(2), 186-194.

Kapur, P.K., Kumar, S., \& Garg, R.B. (1999). Contributions to hardware and software reliability. World Scientific, Singapore.

Kapur, P.K., Panwar, S., Singh, O., \& Kumar, V. (2019). Joint release and testing stop time policy with testing-effort and change point. In Risk Based Technologies (pp. 209-222). Springer, Singapore.

Kapur, P.K., Pham, H., Anand, S., \& Yadav, K. (2011). A unified approach for developing software reliability growth models in the presence of imperfect debugging and error generation. IEEE Transactions on Reliability, 60(1), 331-340.

Kapur, P.K., Pham, H., Gupta, A., \& Jha, P.C. (2011). Software reliability assessment with OR applications. Springer, London.

Kapur, P.K., Singh, J.N., \& Singh, O. (2015). Application of multi attribute utility theory in multiple releases of software. International Journal of System Assurance Engineering and Management, 6(1), 61-70.

Kapur, P.K., Singh, O., Garmabaki, A.S., \& Singh, J. (2010). Multi up-gradation software reliability growth model with imperfect debugging. International Journal of System Assurance Engineering and Management, 1(4), 299-306.

Kapur, P.K., Singh, V.B., Anand, S., \& Yadavalli, V.S.S. (2008). Software reliability growth model with change-point and effort control using a power function of the testing time. International Journal of Production Research, 46(3), 771-787. 
International Journal of Mathematical, Engineering and Management Sciences

Vol. 5, No. 2, 208-224, 2020

https://doi.org/10.33889/IJMEMS.2020.5.2.017

Keeney, R.L. (1971). Utility independence and preferences for multi attributed consequences. Operations Research, 19(4), 875-893.

Li, X., Li, Y.F., Xie, M., \& Ng, S.H. (2011). Reliability analysis and optimal version-updating for open source software. Information and Software Technology, 53(9), 929-936.

Majumdar, R., Shrivastava, A.K., Kapur, P.K., \& Khatri, S.K. (2017). Release and testing stop time of a software using multi-attribute utility theory. Life Cycle Reliability and Safety Engineering, 6(1), 47-55.

Marquardt, D.W. (1963). An algorithm for least-squares estimation of nonlinear parameters. Journal of the Society for Industrial and Applied Mathematics, 11(2), 431-441.

McDaid, K., \& Wilson, S.P. (2001). Deciding how long to test software. Journal of the Royal Statistical Society: Series D (The Statistician), 50(2), 117-134.

Ohba, M., \& Yamada, S. (1984). S-shaped software reliability growth models. In International Colloquium on Reliability and Maintainability, 4th, Tregastel, France (pp. 430-436).

Okumoto, K., \& Goel, A.L. (1980). Optimum release time for software systems based on reliability and cost criteria. Journal of Systems and Software, 1, 315-318.

Pham, H., Nordmann, L., \& Zhang, Z. (1999). A general imperfect-software-debugging model with S-shaped fault-detection rate. IEEE Transactions on Reliability, 48(2), 169-175.

Roy, P., Mahapatra, G.S., \& Dey, K.N. (2014). An NHPP software reliability growth model with imperfect debugging and error generation. International Journal of Reliability, Quality and Safety Engineering, 21(02), 1450008.

SAS, S. (2004). STAT User guide, Version 9.1.2. SAS Institute Inc, Cary, NC, USA.

Wang, J., Wu, Z., Shu, Y., \& Zhang, Z. (2015). An imperfect software debugging model considering loglogistic distribution fault content function. Journal of Systems and Software, 100, 167-181.

Wood, A. (1996). Predicting software reliability. Computer, 29(11), 69-77.

Xie, M., \& Yang, B. (2003). A study of the effect of imperfect debugging on software development cost. IEEE Transactions on Software Engineering, 29(5), 471-473.

Yamada, S., \& Osaki, S. (1987). Optimal software release policies with simultaneous cost and reliability requirements. European Journal of Operational Research, 31(1), 46-51.

Yamada, S., Ohba, M., \& Osaki, S. (1983). S-shaped reliability growth modeling for software error detection. IEEE Transactions on Reliability, 32(5), 475-484.

Zhu, M., \& Pham, H. (2018a). A multi-release software reliability modeling for open source software incorporating dependent fault detection process. Annals of Operations Research, 269(1-2), 773-790.

Zhu, M., \& Pham, H. (2018b). A two-phase software reliability modeling involving with software fault dependency and imperfect fault removal. Computer Languages, Systems \& Structures, 53, 27-42. 